\title{
What Elements Are Needed to Design a Comprehensive Youth Development Programme
}

\author{
H. Pharaoh ${ }^{1, *}$, M. Smith ${ }^{2}$, J. Frantz ${ }^{3}$ \\ ${ }^{1}$ Department of Physiotherapy, School of Health Sciences, University of Kwazulu-Natal, South Africa \\ ${ }^{2}$ Department of Psychology, University of the Western Cape, South Africa \\ ${ }^{3}$ Community and Health Sciences Faculty, University of the Western Cape, South Africa
}

Copyright $\bigcirc 2018$ by authors, all rights reserved. Authors agree that this article remains permanently open access under the terms of the Creative Commons Attribution License 4.0 International License

\begin{abstract}
Interventions to combat engagement in health risk behaviours (HRB) amongst youth produces mixed results. Engagement in HRBs continues to increase with adverse effects on health and well-being. This manuscript reports on a study that attempted to demonstrate the usefulness of Intervention Mapping (IM) in developing contextually relevant programmes with increased buy-in from stakeholders, and the potential to effect behavioural change. The present study used a modified Intervention Mapping (IM) framework that included the first five IM operational steps: 1) Needs assessment (Survey), 2) Identifying performance objectives (Concept Mapping), 3) Methods and strategies (Systematic review), 4) Programme development (Triangulation of all the components in the preceding steps) and 5) Feasibility of the designed programme (Delphi Study). The manuscript demonstrated how the framework enabled the use of multiple methodologies to develop a programme that was methodologically rigorous and empirically grounded. The focus is not on the results of the various steps, but on demonstrating how the steps were applied to facilitate programme development. Ethics clearance was obtained from the UWC Senate Research Committee and all ethics principles observed. The results demonstrate that IM is an appropriate conceptual framework for collaboration that enhances the resultant programme at various levels: theoretical/ conceptual; methodological and practical application/ intervention.
\end{abstract}

Keywords $\begin{aligned} & \text { Life Skills, Youth Development, } \\ & \text { Intervention } \\ & \text { Behaviour }\end{aligned}$

\section{Introduction}

The prevalence of health risk behaviours among youth is on the increase both internationally and nationally, and is becoming a public health concern. Some of these preventable health behaviours may be contributory causes of morbidity and mortality [1]. Data concerned with health risk behaviour published worldwide further underscored the importance of and urgency for immediate intervention [2]. South African National Youth Risk Behaviour Surveys reported engagement in the following risky behavior: unsafe sexual practices $(38 \%)$, obesity $(20 \%)$, suicide attempts $(21 \%)$ and substance abuse $(30 \%)[3,4]$. Reddy et al. [4] reported that that engagement in health risk behaviours have increased from the initial survey estimates in the lifetime and current engagement from the 2002 survey. In addition, first time use is increasingly reported at younger ages with increased vulnerability during transitions such as moving to secondary school [5]. Thus there is a need to develop programmes aimed at learners in grades 8-10 aged 13-16. Reddy et al. [4] also reported unchanged estimates in current use or engagement in health risk behaviours among youth despite major resources being channeled towards education and interventions. Thus engagement in health risk behaviour among young people continues to be a problem and interventions have mixed results. Behavioural change is not taking place effectively to combat health risk behaviour [6]. Literature highlighted some of the challenges and shortcoming that have contributed to the limited success. Specific recommendations for successful programming include the use of multi-behavioural programmes, active youth participation, a theoretical grounding, and the use of specific content such as skills education [7]. It has been found that successful interventions need to be contextually relevant, have increased buy-in from all stakeholders, and have the potential to effect behavioural change not only at individual level but also at community level. Growing evidence suggests that effective programmes orientated towards individual health behaviours require a multifaceted approach to helping people adopt, change, and maintain healthful behaviour [8]. Cummings [9] recommended that family and school contexts as well as individual characteristics should be taken into account when developing intervention programmes. Similarly, intervention programmes should be informed by research 
knowledge and evidence of best practice. It is very important to adequately focus on changing behaviour in a community or certain environment when designing youth development programs that addresses health and social care. Researchers need to make sure that ways are found to incorporate all the elements that are needed for that change to take place. Interventions cannot only be implemented for those at risk should also incorporate every stakeholder within the community that interacts within the changing environment. Incorporating all stakeholders plays a pivotal role in attempting to effect change and increase buy-in [2]. Thus there is a need for comprehensive approach to designing interventions that includes stakeholder consultation, research evidence and interdisciplinary collaboration. This article reports on the steps taken to design a diverse comprehensive youth development programme for grade 8-10 learners aged between 13-18 years in order to equip learners with the skills to change engagement in health risk behaviour. The aim of the article is not to report the results of the respective phases per se, but to present the overarching conceptual approach to programme development.

\section{Framework for the Study}

This study used a modified Intervention Mapping (IM) as the framework to guide the process of programme design. The IM framework is a stepwise approach to describe the planned process for theory and evidence-based development, implementation and evaluation of health promotion interventions [10]. Bartholomew, Parcel and Kok [11] developed IM to assist health promoters to develop the best possible intervention using planning, research and theory as key components. This approach allows for an interdisciplinary focus as it aims to ensure that all stakeholders with a common vision address a common problem. The study was therefore conceptualised as a modified intervention mapping process consisting of five phases aligned with the general framework including, 1) Needs assessment; 2) Identifying performance objectives, 3) Methods and strategies, 4) Programme development, and 5) Feasibility. Each phase was developed independently with its own methodological elements. The findings from each phase were used to inform the next phase, as illustrated by Figure 1 below.

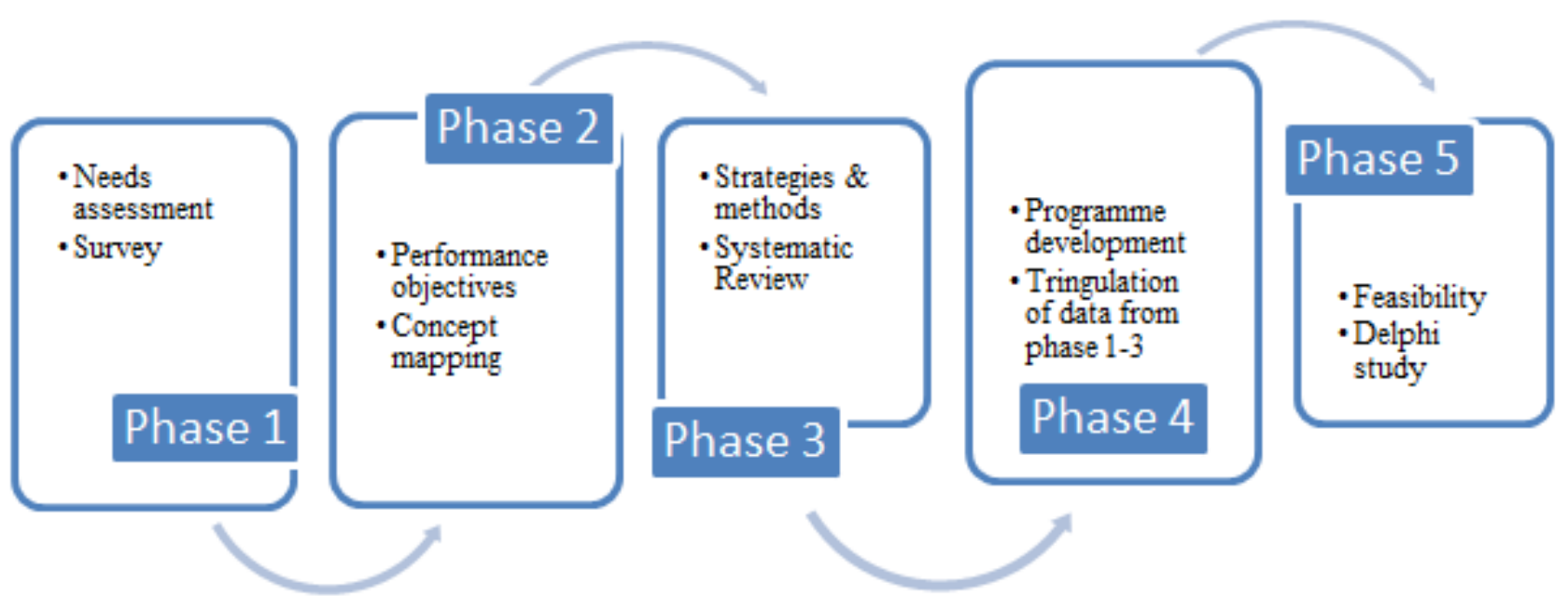

Figure 1. Brief overview of overarching methodology 


\section{Phase 1: Needs Assessment}

The aim of this step was to assess the health problem, its related behaviour and environmental conditions, and associated determinants for the at-risk populations. The outcome of this first step is a description of engagement in health risk behaviours in the target population (health problem), the impact on quality of life (consequence), behavioural and environmental determinants of behaviour. To this end, a survey was conducted with 1027 grade 8-10 learners to obtain baseline information about the health risk behaviours they engaged in, and the extent to which learners managed personal situations. Survey methodology was appropriate for gathering cross-sectional information from a large sample group in a cost-effective manner. The survey included the following instruments: 1) a self-constructed demographic questionnaire, 2) Youth Risk Behaviour Surveillance Survey (YRBSS) [12], 3) Life Effectiveness Questionnaire (LEQ) [13]. Descriptive statistics, specifically frequencies and percentages, were used to summarise demographic data, engagement in risk behaviours, and life skills domains. The data from the (YRBSS) and LEQ survey were subjected to frequency distributions, cross tabulations and Chi-square tests for significant differences. The relationship between life skills (Life Effectiveness Questionnaire data) and the most frequently reported health risk behaviours for this sample was assessed using multiple regression analysis. For a comprehensive report on the results of this phase, consult Pharaoh Unpublished Thesis [14]. In short, the results concurred with South African national surveys of youth risk behaviour regarding the nature and extent of engagement in health risk behaviours. The results from this survey underscored that engagement in health risk behaviours such as smoking, drinking, drug use, sexual activity and physical inactivity is still increasing and provided empirical support for gendered patterns in how youth engage in smoking, drinking and risky sexual activity that in turn requires programming initiatives to be sensitive to the impact of gender. The most frequently reported health risk behaviours in this sample were smoking, drinking, drug use, and sexual activity. As mentioned before, the survey data was also used to assess the relationship between life skills (Time management, Achievement, Emotional control, Social competence, Active initiative, Self-confidence, Intellectual flexibility and Task leadership) as measured by the LEQ and engagement in high frequency health risk behaviours. Most intervention programmes include life skills training based on the assumption that it will increase the ability to delay or avoid engagement in HRBs. The data was used to test the converse assumption that engagement in HRBs was a function of lifeskills or lack thereof. The results of the regression analyses were published in Pharaoh, Frantz and Smith [15]. The results indicated that reported capacity in identified life skills domains could significantly predict engagement in high frequency HRB (smoking, drinking, drug use, sexual activity). Thus the extent to which youth possess (or lack) life skills can significantly predict whether health risk behaviours are engaged in. Thus in the first phase, the authors determined the scope of engagement in HRB for the intended population group and was able to provide empirical support for 1) increased engagement, 2) gendered patterns of engagement and 3) predictive relationship between lifeskills and engagement in high frequency HRBs.

\section{Phase 2: Identifying Performance Objectives}

The second phase of IM is the definition of programme (change) objectives based upon scientific analyses of health problems and problem-causing factors. To this end, concept mapping was adopted to achieve the objectives of this phase. Concept maps are graphical tools for organising and representing knowledge [16]. They include ideas or concepts usually enclosed in circles or boxes of some type, and relationships between concepts or propositions, indicated by a connecting line between two concepts. Words on the line specify the relationship between the two concepts. Concepts are defined as a perceived regularity in events or objects, or records of events or objects, designated by a label [17]. The present study used a modified version of concept mapping to identify qualitative concepts from focus groups and interviews with a purposive sample of 32 high school learners aged between 13-18 years, 4 high school teachers, a district representative involved in school based programmes, and two community leaders involved in youth development from the target community. Based on the gendered pattern identified in Phase One, the learners were divided into three groups including a male group $(n=10)$, a female group $(n=10)$ and a mixed-gender group $(n=12)$. During the preparation step, the authors read extensively in the focus area of the study to formulate the focus prompts for a focus group discussion with various stakeholders. The second step incorporated data collection to generate participant responses to the focus prompts. The analysis step incorporated thematic analyses of transcripts and the distilling of concepts generated from the participants. Recurrent themes were identified and collated into four subsidiary concept maps that were graphically represented in one meta-figure or concept map.

The results of this step were published in Pharaoh, Frantz and Smith [18] provided a more in-depth discussion of the results. The results included four themes: 1) HRBs that learners engage in, 2) Perceived reasons why youth engage in HRBs, 3) Places of exposure to health risk behaviour, and 4) Specific content or focuses. Each theme further represents concepts and relations between propositions of stakeholders' perceptions about what that the content of proposed programmes aimed at combating HRBs amongst youth should include. Figure 2 below represents the resultant concept map. 


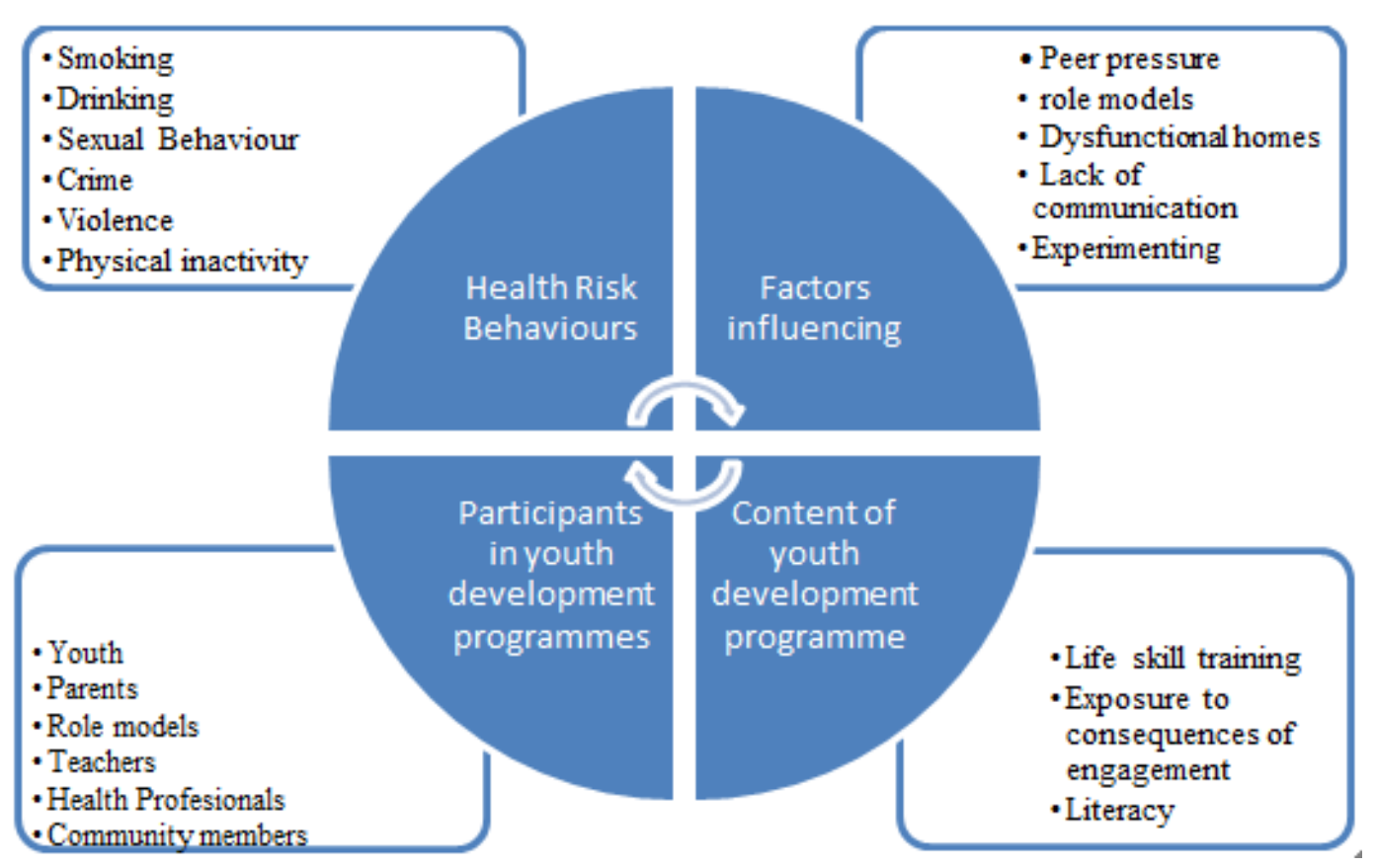

Figure 2. Themes and subthemes

The above concept map contains valuable information about what stakeholders thought intervention programmes should include. This process provided stakeholders an opportunity to actively collaborate in programme development that in turn would likely increase their buy-in. The consultative process also provided programme developers and by extension subsequent facilitators credibility with the intended community and target group. Thus this phase underscored how participatory methods can be used to facilitate stakeholder participation that strengthens buy-in and provides a more collaborative approach to identifying performance or change objectives.

\section{Phase 3: Methods and Strategies}

This phase of IM was dedicated to the selection of theory-based intervention methods and practical applications to change determinants of health-related behaviour. To this end, Systematic review methodology was adopted to execute this phase of the present study. Systematic review enabled the researcher to consolidate the body of literature reporting on intervention content for the target group through a filtration process. The identified literature is evaluated for methodological quality and articles that exceed a pre-established threshold score are included in the final summation. Systematic reviews follow a rigorous and explicit methodology that can provide a reliable basis for decision making about the content that was included in interventions reported in good quality research. A total of 959 potential titles were identified of which 15 articles were included in the final summation. Data were extracted from the final summation about 1) general description of studies, 2) Intervention content, 3) empirical evidence of the interventions and 4) theoretical orientations of interventions. The results of this phase have been reported comprehensively in the unpublished thesis of Pharaoh [14] and are currently under review for publication.

From the systematic review it emerged that there was good quality research available that provided empirical support based on methodological rigor for a theoretical foundation for the programme. The core findings suggested that interventions seem to be most effective when they widen the scope and include other aspects such as life skills, sport, and parental education/involvement [19]. The studies with the best results were those that included more than one of these additional elements mentioned above [20, 21]. The addition of sport was reportedly particularly powerful at increasing positive, more realistic attitudes and perspectives regarding the self and others [22]. These elements in turn were successful at reducing health risk behaviours $[23,24]$. Parental involvement was reportedly effective at reinforcing positive effects of the intervention and maintaining long-term results [25]. Life skills education was particularly effective with females and at helping learners in poorer areas cope with community stressors $([24,20]$. Generally the interventions targeting younger intermediate students were more effective than interventions targeting older intermediate and senior students. Some studies also suggest that interventions conducted with non-drug users were more effective than with drug users. Interventions which used trained professionals as facilitators were also generally more successful [26]. Moreover, these findings clearly indicate that drawing from different professional expertise and skill sets provided more robust results as evidenced by the effect sizes and significance levels [14]. This would be consistent with the underlying tenets of inter-professional education 
and collaboration. The findings of this phase provided valuable information from published good quality research that provided insights into important theoretical aspects for consideration in programme development with empirical evidence. Thus it was noted that there is a gendered and cohort (age) response to interventions, professional facilitation was recommended, and that multiple elements are more effective including parental involvement, life skills and sport. The reported efficacy with non-user groups in delaying onset was duly noted and considered in the expansion of the target group.

\section{Phase 4: Programme Development}

The aim of this phase of the IM was to triangulate the data from previous phases that would inform the design of a youth development programme. The first three phases in the IM built upon each other in gaining information that would ultimately form the basis of the designed programme. The resultant programme would include a description of the scope, content and sequence of the components of the intervention, completed programme materials, and programme protocols. This phase demands careful reconsideration of the intended programme participants and the programme context. The figure below illustrates all the information gathered to design the first draft of the youth development programme inclusive of the proposed content. As mentioned before, the aim of this paper is not to report on the content of the programme but the process flow resulting in the programme within an IM framework.

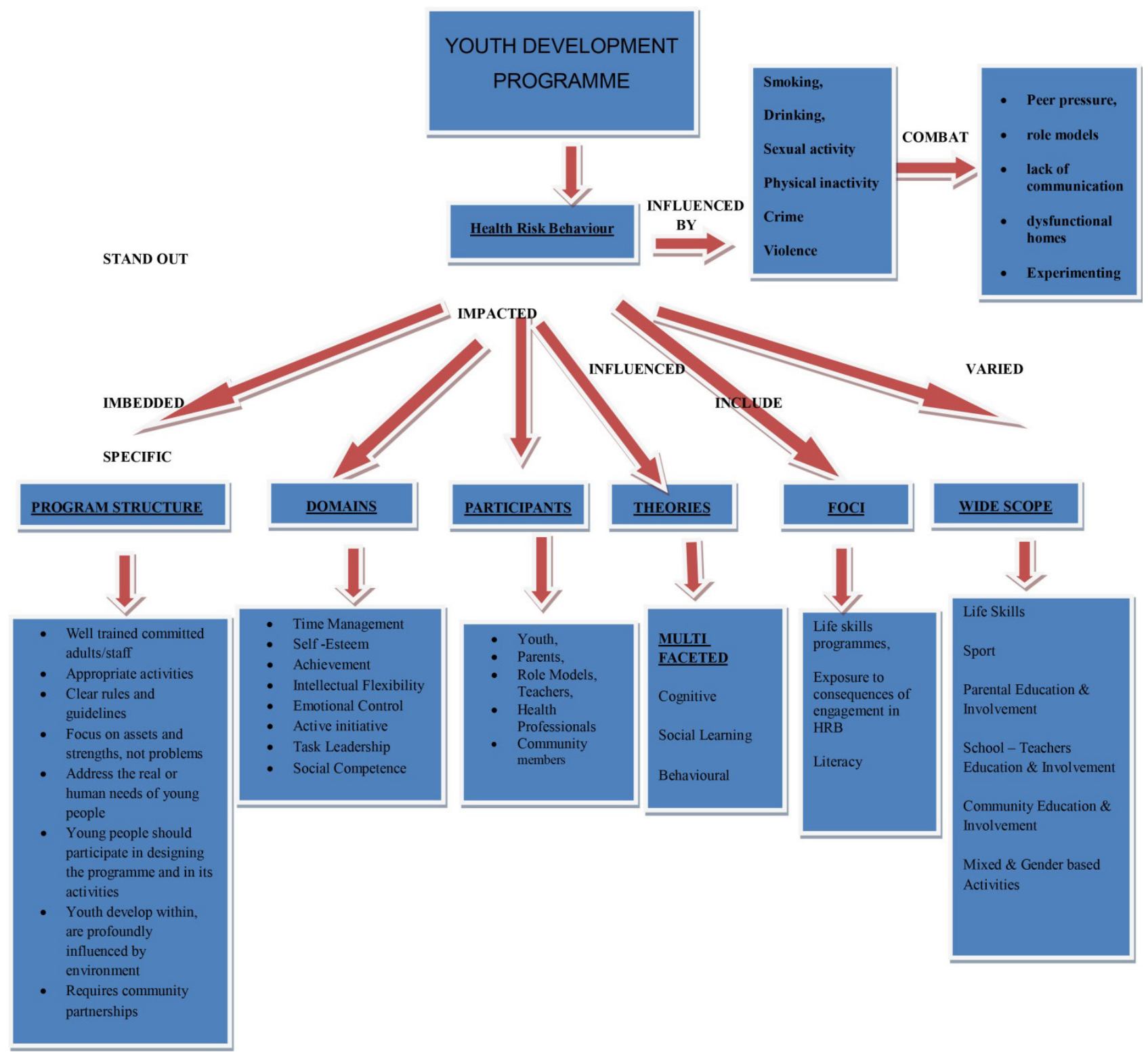

Figure 3. Design Structure 


\section{Step 5: Feasibility}

This phase used the Delphi method to assess the feasibility of the resultant youth development programme. The Delphi method is an iterative process in which a panel of experts responds confidentially to a stimulus document. The feedback of the panel members is engaged with and the stimulus document is refined in successive rounds until consensus is reached on the final product. Young and Jamieson [27] asserted that the Delphi method is appropriate for gaining expert opinion and consensus around important aspects pertaining to the design of an intervention as described in the present study. For the purposes of this study a panel of 24 experts in several professions were purposive selected, as well as individuals who were steeped in community development work. The composition of the panel of experts made it possible for the researcher to capture various expertises pertaining to youth development and health risk behaviour interventions. The panel gave input as to: (i) the scope of the programme, (ii) the content of the programme, (iii) the approaches to be used in the implementation of the programme, (iv) the implementation strategy of the programme, (v) the resources needed for the implementation of the programme and (vi) the costing involved to implement the designed programme. Quantitative data from the Delphi study was summarized using measures of central tendency [28]. The qualitative data was analysed thematically under the components of the programme. The results of this phase was comprehensive discussed in Pharaoh unpublished thesis [14]. For the purposes of this manuscript, the focus was on demonstrating the iterative process. The Delphi study was conducted in two rounds. The experts rated their degree of agreement on a scale of 0 (no agreement) to 5 (full agreement). Thus higher scores indicated higher agreement. The percentage of agreement was then calculated across the panel for each item on the stimulus document and a score of $75 \%$ or more was considered as consensus. During Round 1, consensus was reached on almost $80 \%$ of the items. The remainder of the items were revised and presented to the panel in a second round. Full consensus was reached at the completion of Round 2. The final consensus enabled the researcher to gain an understanding from experts as to the feasibility of the components of the programme, as well as the overall programme. Thus the draft programme was accepted as a feasible programme for the target group. The resultant programme is therefore illustrated in Figure 4 below:

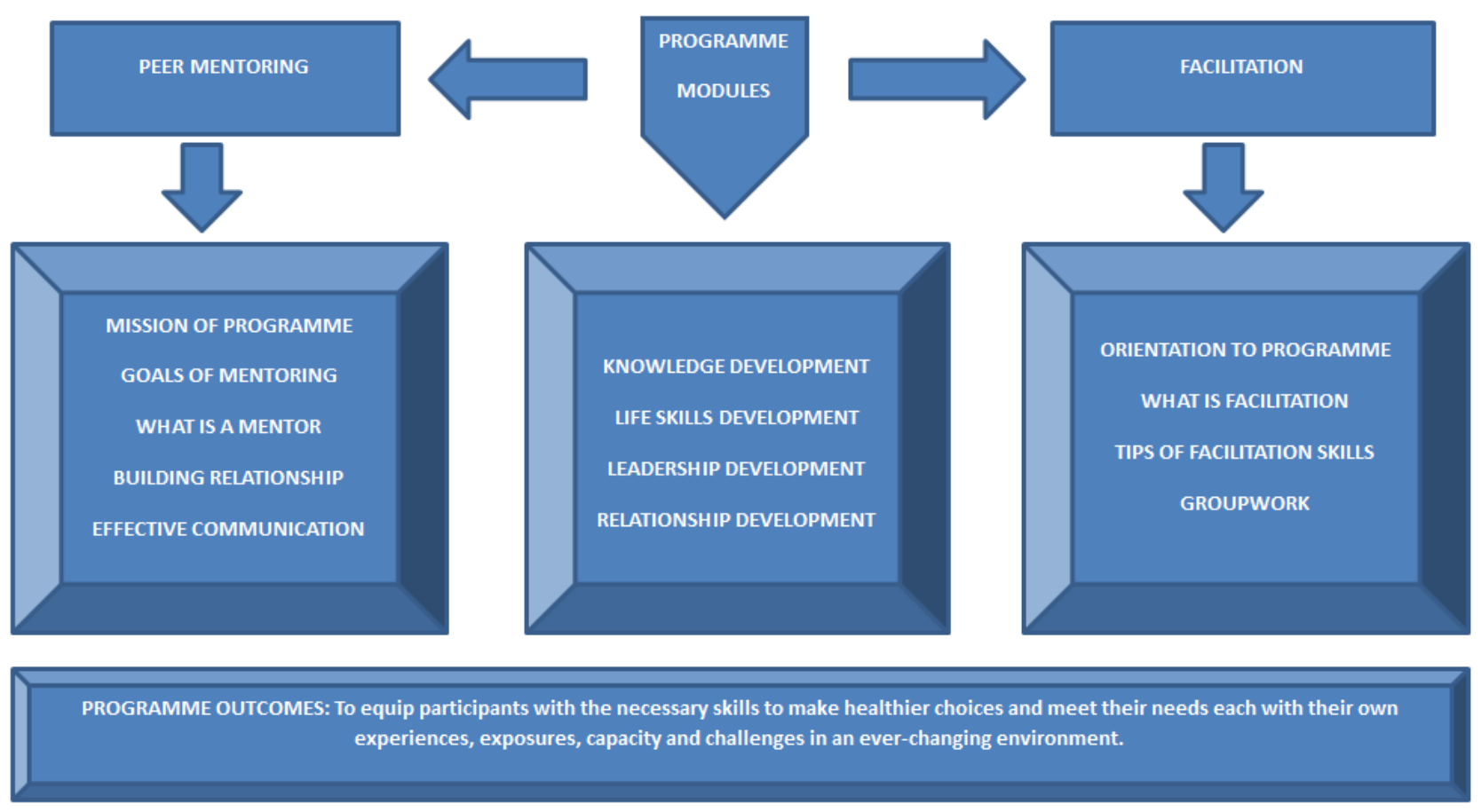

Figure 4. Developing Youth 4 Paarl (DY4P) 
The panel made the following additional recommendations:

- Scaffolding of the programme into specific ageand gender-based activities;

- Prioritizing the contextual relevance of the programme so that it remains flexible enough to be sensitive to the diverse needs and background of its participants;

- Training manuals and protocol for facilitators in order to ensure that facilitators are equipped to deal with the challenges that will emerge throughout the programme.

The draft programme was designed for learners in the intermediate phase of secondary school (grade 8-10/ aged 13-16). The first recommendation meant that the programme had to be revised such that it could be presented to age or grade cohorts. The second recommendation raised some design elements in the programme to allow for optimal flexibility. The design process resulted in a draft programme (stimulus document) that was targeted for the identified community, but generalization to other settings would require an adaptation protocol. The third recommendation challenged the proposed use of professional staff exclusively. Often community-based projects are hamstrung by funding and the recommendation of a training protocol would ensure that the programme was not dependent on the availability of professional staff and reduce overheads associated with employing professional staff. This would then contribute to capacity building and increased sustainability. These revisions were made in addition to the consensus on the proposed programme. The adoption of these recommendation meant that a major revision of the base programme would be required.

\section{Concluding Comments}

The limitations in current programmes for reducing or delaying engagement in health risk behavior has been well-documented. Increased success for intervention programmes are linked to design processes that are consultative, stakeholder buy-in and empirically grounded development. This manuscript reports on the feasibility of using a modified Intervention Mapping to design a youth development programme for learners between 13 and 16 years old. Intervention Mapping facilitated a process that was a) grounded on contextual sensitivity and relevance, b) theoretical and empirical grounding, c) consultative with stakeholders, and feasibility testing. The process contributed to developing a programme that Finally the following elements were identified that drive the design of a comprehensive YDP: a) Framework to guide the process; b) understanding the factors that influences health risk behaviour; c) creating a templet that depicts what is needed to design the YDP that is specific to the participants and d)
Testing the feasibility of the YDP among experts and relevant stakeholders. The resultant programme was comprehensive and incorporated a multi-faceted approach based on the current health risk behaviour that the youth engage in. Active stakeholder engagement contribute to the diversity of the programme and increases buy-in. The IM process expands the stakeholder groups to include the target group, caregivers, community and government workers, as well as professionals, academics and researchers. The use of IM facilitated a process in which collaboration enhances the resultant programme at various levels: theoretical/ conceptual; methodological and practical intervention. At a theoretical level, various points of references could be used to develop a multi-dimensional understanding of the problem and context. At a methodological level the use of participatory methods and secondary research alongside traditionally empirical or cross-sectional methodologies resulted in a stronger foundation for the programme that in turn increased the likelihood of being effective. IM provided an overarching umbrella or conceptual framework at a design level that enabled these often perceived non-complimentary methods to be used in tandem. At a practical or intervention level, the input from various professional vantage points enabled the programme to be more relevant for the context in which it was going to be implemented.

\section{REFERENCES}

[1] Grunbaun, J., Kann, L., Kinchen, A., \& al, e. (2001). Youth risk behavior surveillance - United States. Journal of School Health, 2002(72), 313-328.

[2] WHO. (2010). Global status report on non-communicable deseases. 1 Chronic diseases - prevention and control. 2. Chronic diseases - epidemiology. 3. Chronic disease mortality. 4. Cost of illness. 5. Delivery of health care. World Health Organization.

[3] Reddy, S., Panday, S., Swart, D. J., Amosun, S., \& James, S (2002). Umthente Uhlaba Usamila - The South African Youth Survey . Cape Town: South Africa Medical Research Council.

[4] Reddy, S., James, S., Sewpaul, R., Koopman, F., Funani, N., Sifunda, S., Omardien, R. (2010). Umthente Uhlaba Usamila- The South African Youth Risk Behaviour Survey. Cape Town: South African Medical Research Council

[5] Lester, L., \& Cross, D. (2015). The Relationship Between School Climate and Mental and Emotional Wellbeing Over the Transition from Primary to Secondary School. Psychology of Well-Being, 5(1), 9. http://doi.org/10.1186/s13612-015-0037-8

[6] Zulkifi, S., \& Wong, Y. (2002). Knowledge, attitudes and beliefs related to HIV/AIDS among adolescents in Malaysia. The Medical Journal of Malaysia, 57(1), 3-23.

[7] Villa-Torres, L \& Svanemyr, J (2014). Youth's Right to 
Participation and Promotion of Youth Leadership in the Development of Sexual and Reproductive Health Policies and Programs. Journal of Adolescent Health 56 (2015) S51eS57

[8] Hawkins, J.D., Catalano, R.F. \& Arthur, M.W. (2002). Promoting science-based prevention in communities. Addictive Behaviors. 27:951-976. [PubMed].

[9] Cummings, M. E., Goeke-Morey, M. C., \& Papp, L. M. (2003). Children's responses to everyday marital conflict tactics in the home. Child Development, 74, 1918-1929.

[10] Bartholomew, K., Parcel, G., Kok, \& Gottlieb, N. (2001). Intervention Mapping: Designing Theory and Evidence-Based Health Promotion Programs. Mountian View : CA: Mayfield

[11] Bartholomew, L., Parcel, G., \& Kok, G. (1998). Intervention Mapping: a process for designing theory and evidence-based health education programs. Health Education and Behavior, $25,545-563$

[12] YRBSS: Youth Risk Behavioural Surveillance System 2002 (On Line). Available: www.cdc.gov/health youth/yrbss (2010, March 07).

[13] Neill, J. T., Marsh, H. W., \& Richards, G. E. (2003). The Life Effectiveness Questionnaire: Development and psychometrics. Unpublished manuscript, University of Western Sydney, Sydney, NSW, Australia.

[14] Pharaoh, H.G (2015). Unpublished Thesis. https://etd.uwc.ac.za/bitstream/handle/11394/4297/pharaoh _h_phd_chs_2014.pdf? sequence $=1 \&$ is Allowed $=y$

[15] Pharaoh, H., Frantz, J. \& Smith, M. (2011). Life skills as predictors of engagement in health risk behaviours: A survey of secondary school learners. African Journal for Physical, Health Education, Recreation and Dance, June (Supplement), 70-81.

[16] Novak, J.D \& Cañas, A.J (2008). Institute for Human and Machine Cognition Pensacola Fl, 32502. www.ihmc.us Technical Report IHMC CmapTools 2006-01 Rev 2008-01

[17] Engelbrecht, A., Mintzes, J., Brown, L., \& Kelso, P. (2005). Probing Understanding in Physical Geology Using Concept Maps and Clinical Interviews. Journal of Geoscience Education, 53, 263-270.

[18] Pharaoh H, Frantz J \& Smith M. Concept Mapping: Stakeholders ' perception about what should be included in interventions programmes aimed at reducing engagement in health risk behaviour amongst youth. African Journal for Physical, Health Education, Recreation and Dance (AJPHERD), October 2014 (Supplement) pp. 44-58
[19] Soper, A. C., Wolchik, S. A., Tein, J.-Y., \& Sandler, I. N. (2010). Mediation of a preventive intervention's 6-year effects on health risk behaviors. Psychology of Addictive Behaviors, 24(2), 300-310. Spera, C. (2005). A review of the relationship among parenting practices, parenting styles, and adolescent school achievement. Educational Psychology Review, 17(2), 125-146.

[20] Botvin, G. J., Griffin, K. W., and Nichols, T. R. (2006). Preventing youth violence and delinquency through a universal school-based prevention approach. Prevention Science, 7, 403-408.

[21] Spoth, R.; Greenberg, M.; and Turrisi, R. Preventive interventions addressing underage drinking: State of the evidence and steps toward public health impact. Pediatrics 121:S311-S336, 2008. PMID: 18381496

[22] Werch, C., Moore, M., Di Clemente, C.C., Owen, D.M., Jobli, E. \& Bledsoe, R. A. (2003). Sport-based intervention for preventing alcohol use and promoting physical activity among adolescents. Journal of School Health. 73(10):380388. [PubMed]

[23] Eisen, M.; Zellman, G.L.; and Murray, D.M. Evaluating the Lions-Quest "Skills for Adolescence" drug education program: Second-year behavior outcomes. Addict Behav 28(5):883-897, 2003

[24] Huang, Y.F., Kuo, H.S., Lew-Ting, C.Y., Tian, F., Yang, C.H., Tsai, T.I., Gange, S.J. \& Nelson, K.E. (2011). Mortality among a cohort of drug users after their release from prison: an evaluation of the effectiveness of a harm reduction program in Taiwan. Aug; 106(8):1437-45. doi: 10.1111/j.1360-0443.2011.03443.x. Epub 2011 May 12.

[25] Visser, L., de Winter, A.F., Veenstra, R., Verhulst, F.C. \& Reijneveld, S.A. (2012). Alcohol use and abuse in young adulthood: Do self-control and parents' perceptions of friends during adolescence modify peer influence? The TRAILS study. Addictive Behaviours, 12: 2841-2846

[26] Smith, E. A., Swisher, J.D. \& Vicary, J et al. (2004). "Evaluation of Life Skills Training and Infused-Life Skills Training in a Rural Setting: Outcomes at Two Years," Journal of Alcohol and Drug Education, Vol. 48, No. 1, 2004, pp. 51-70.

[27] Young, S. J., \& Jamieson, L. M. (2001). Delivery methodology of the Delphi: A comparison of two approaches. Journal of Parl Recreation Administration, 19(1), 42-58

[28] Gravetter, F. J., \& Wallnau, L. B. (2000). Statistics for the behavioral sciences (5 ed.). Belmont: Wadsworth-Thomson Learning. 